\title{
Efecto de la relación enzima-sustrato en la hidrólisis enzimática de lactosuero bovino por Alcalasa ${ }^{\circledR} 2.4 \mathrm{~L}$
}

\author{
Omar A. Figueroa (1), Sandy P. Peñaloza(2), Emil E. Mejía(2) y José E. Zapata(3)* \\ (1) Facultad de Ingeniería, Dpto. de Ingeniería Agroindustrial, Universidad de la Guajira, Km 5 Vía Maicao, Riohacha, - \\ Colombia. (e-mail: omfimo22@gmail.com) \\ (2) Facultad de Ingeniería, Dpto. de Ingeniería Agroindustrial, Univ. Popular del Cesar, Diagonal 21 No. 29-56 \\ Valledupar-Colombia. (e-mail: enriq-9257@hotmail.com; sandyppenaloza@unicesar.edu.co) \\ (3) Facultad de Ciencias Farmacéuticas y Alimentarias, Dpto. de Alimentos, Univ. de Antioquia, Calle 70 \# \\ 52-21, Apartado aéreo 1226, Medellín, Colombia. (e-mail: edgar.zapata@udea.edu.co)
}

* Autor a quien debe ser dirigida la correspondencia

Recibido Sep. 9, 2019; Aceptado Oct. 29, 2019; Versión final Ene. 18, 2020, Publicado Jun. 2020

\section{Resumen}

El objetivo de este estudio fue analizar la capacidad antioxidante (medida con FRAP y ABTS) y el potencial quelante de hierro a través del tiempo en distintas condiciones de enzima y sustrato. Se realizó la hidrólisis enzimática de lactosuero bovino en polvo, en un reactor por lote con capacidad de 0,5 L, y temperatura constante. Los hidrolizados con mayor actividad fueron separados de acuerdo a su tamaño molecular a razón de $>100,10-100,<10$ y $<3 \mathrm{KDa}$ para verificar la capacidad antioxidante aportada por cada uno de ellos. Los datos obtenidos mostraron que la actividad antioxidante es mayor a menores concentraciones de enzima, mientras que a sustratos bajos el potencial de fracciones peptídicas con capacidad para neutralizar cationes $\mathrm{ABTS}^{+}$es bajo. El incremento en el grado de hidrólisis favorece la actividad antioxidante medida por el método ABTS, así como la actividad quelante de hierro de los hidrolizados.

\section{Effect of the enzyme-substrate relationship on the enzymatic hydrolysis of bovine whey by Alcalasa ${ }^{\circledR} 2.4 \mathrm{~L}$}

\begin{abstract}
The main objective of this study was to analyze the antioxidant capacity (measured with FRAP and ABTS) and the chelating potential of iron over time at different enzyme and substrate conditions. Enzymatic hydrolysis of bovine whey powder was performed in a batch reactor with a capacity of $0.5 \mathrm{~L}$ and constant temperature. The most active hydrolysates were separated according to their molecular size at a rate of $>100,10-100,<10$ and $<3 \mathrm{KDa}$ to verify the antioxidant capacity contributed by each of them. The data obtained showed that antioxidant activity is higher at lower enzyme concentrations. But, the potential for peptide fractions with the ability to neutralize $\mathrm{ABTS}^{+}$cations is low at low substrates. The increase in hydrolysis degree favors the antioxidant activity measured by the ABTS method and the chelating activity of hydrolyzed metals.
\end{abstract}




\section{INTRODUCCIÓN}

El lactosuero bovino (LSB) tiene un valioso valor proteico representado principalmente en $\beta$-lactoglobulina ( $\beta$ LG) y a-lactoalbúmina (Hinrichs et al., 2004). Los hidrolizados enzimáticos de proteína de lactosuero han sido ampliamente usados como ingrediente bioactivo y nutricional en productos para salud y alimentos (Marshall, 2004). Es conocido que péptidos producidos tras hidrólisis tienen potencial biológico específico, en el que resalta la actividad antioxidante (Liu et al., 2010). Esta actividad se asocia con la capacidad de los péptidos para inhibir la peroxidación lipídica y la capacidad de quelar iones metálicos (Sarmadi e Ismail, 2010). Los antioxidantes sintéticos convencionales (BHA, BHT y n-propilo galato) muestran una fuerte actividad contra varios sistemas oxidantes, pero presentan estricta regulación en su uso, por su relación con riesgos potenciales a la salud (Sabeena et al., 2014). Por lo tanto, es tendencia en el mundo, la producción y el empleo de productos naturales antioxidantes cuya actividad se lleve a cabo no solo en los alimentos envasados, sino también una vez el alimento ha sido ingerido, dado que ha sido probada la relación entre la ingesta de compuestos antioxidantes y la prevención de enfermedades (Gómez et al., 2013En tal sentido, es relevante la realización de este tipo de estudios, tendientes a definir condiciones apropiadas de hidrolisis en el estudio del potencial bioactivo, sobre todo cuando se emplean matrices como subproductos industriales, en tanto se avanza en el análisis de potencialidades se productos con poco valor económico.

Diferentes estudios han establecido la relación existente entre la actividad biológica de los péptidos y su peso molecular. En particular las fracciones de péptidos con pesos moleculares entre 1 - 4kDa, serían las más interesantes para usos nutricionales y/o farmacéuticos (Saidi et al., 2014). Tanto la ultra como la nanofiltración son tecnologías utilizadas con relativo éxito para purificar péptidos con efectos bioactivos de hidrolizados de proteínas lácteas, soya y sustratos vegetales (De Castro y Sato, 2015). La reacción de hidrolisis enzimática de proteínas posee gran complejidad debido principalmente a: i) la naturaleza variada y muchas veces desconocida de los sustratos (estructuras primarias y terciarias de proteínas distintas), ii) la formación de nuevos sustratos de hidrólisis a medida que la reacción avanza, iii) liberación de péptidos cortos y aminoácidos libres que causan inhibiciones serias. iv) inactivación de la enzima en el tiempo (Valencia et al., 2015), y v) efectos asociados a la variada reactividad de los enlaces en relación con la enzima usada, así como la accesibilidad de estas a sitios específicos de reacción (estructura terciaria y cuaternaria de las proteínas) (Fernández y Riera, 2013).

En realidad, si se quiere producir fracciones peptídicas de interés antioxidante, es importante reconocer que estos fenómenos propios de la hidrólisis proteica afectan los productos y que éste efecto se ve influenciado no solo por condiciones de operación ampliamente estudiadas como el pH y la temperatura, si no por los niveles de sustrato y enzima que se usen. Por ejemplo, aumentar la concentración de sustrato, reduce el consumo de agua y energía del proceso. Sin embargo, produce valores bajos de GH final, lo que es aún más complejo, impacta el mecanismo de la hidrólisis (Butré et al., 2012), lo que puede significar formación de péptidos distintos en cada tiempo con expresiones de capacidad antioxidantes variada. En este estudio se propuso analizar el efecto del sustrato y la enzima en la expresión antioxidante de LSB y sus hidrolizados empleando Alcalasa ${ }^{\circledR} 2,4 \mathrm{~L}$, a través de técnicas de laboratorio, capacidad de reducción férrica (FRAP) y el método azinobis 3-etilbenzotiazolina-6-ácido sulfónico (ABTS), empleando el análisis de varianza (ANOVA) de dos factores con medidas repetidas en el tiempo de hidrólisis. Así se analizaron de forma independiente los niveles de sustrato y enzima y su relación con la capacidad antioxidante en el tiempo, lo que implica un análisis con distintos grados de hidrolisis (GHs). Adicionalmente se determinó la capacidad para captar metales como el hierro de los hidrolizados de LSB a distintos GHs.

\section{MATERIALES Y MÉTODOS}

A continuación, se describen elementos relacionados con los controles de los sistemas de reacción, los métodos analíticos realizados y el diseño de experimentos empleado.

\section{Sistema de reacción}

La hidrólisis se llevó a cabo en un reactor de vidrio de $0,5 \mathrm{~L}$ de capacidad, con chaqueta de circulación de agua para regular la temperatura, conectada a un baño de circulación con regulador termostático MX07R-20 (Thomas Scientific, EE. UU $\pm 0.07^{\circ} \mathrm{C}$ ). Para el control del pH y registro de la temperatura de la reacción, se usó un electrodo combinado de vidrio con diafragma esmerilado fijo, conectado a un titulador automático Titrando 842 (Metrohm, Suiza), operado por ordenador (software Tiamo 1.2.1), como lo muestra la Fig. 1. El medio de reacción fue constantemente agitado utilizando un agitador magnético 801 (Metrohm, Suiza) con velocidad de $400 \mathrm{rpm}$. La temperatura y el pH en el sistema se mantuvieron contante con valores de $61^{\circ} \mathrm{C}$ y 9 , respectivamente.

\section{Materiales}

Se empleó lactosuero pulverizado de leche de bovino, adquirido de un proveedor comercial en la ciudad de Bogotá-Colombia, con un contenido de proteína declarado de $12 \%$. Este dato fue corroborado mediante el 
método de Kjeldahl (AOAC 2005, 954.01), empleando 6,38 como factor de conversión para la estimación del contenido proteico. La enzima corresponde a una endoproteasa de Bacillus licheniformis del preparado comercial Alcalasa ${ }^{\circledR} 2.4 \mathrm{~L}(2,4 \mathrm{AU}-\mathrm{A} / \mathrm{g})$ de grado alimentario (Novozymes, Dinamarca).

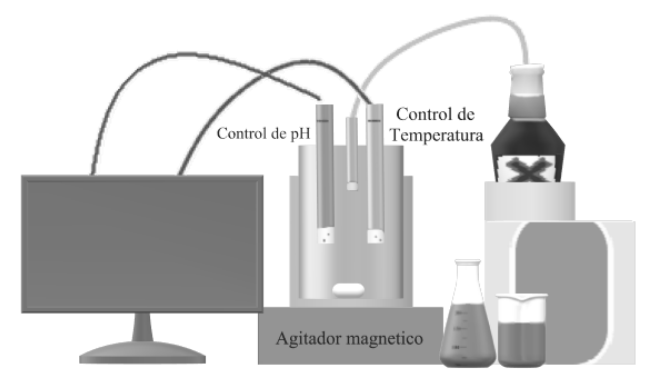

Fig.1. Esquema des sistema de reacción de hidrolisis utilizado en el experimento.

\section{Proceso de hidrolisis enzimática}

La reacción se llevó a cabo mediante hidrolisis enzimática controlado con el método pH-stat, reconocido como el más empleado en hidrólisis enzimática por su simplicidad (Rutherfurd, 2010), el cual consiste en mantener el pH constante mediante la adición de base o ácido diluido. Para este caso, por ser una reacción alcalina se utilizó $\mathrm{NaOH} 1 \mathrm{M}$. En principio, la base agregada para mantener constante el pH, neutraliza únicamente los protones liberados durante la ruptura del enlace amida de las proteínas y los péptidos a pH alcalino, que son sustituidos por el catión de la base; de este modo los protones generados por la hidrólisis son equivalentes a los moles de la base añadida (Adler-Nissen, 1986). La estimación del GH se hizo empleando la ecuación (1).

$$
\mathrm{GH}=\frac{\mathrm{h}}{\mathrm{h}_{\mathrm{tot}}} \times 100=\frac{\mathrm{V}_{\mathrm{NaOH}} \times \mathrm{N}_{\mathrm{b}}}{\mathrm{Mp} \times a \times \mathrm{h}_{\text {tot }}} \times 100
$$

Dónde: $\mathrm{h}=$ Número de enlaces peptídicos hidrolizados; $\mathrm{H}_{\text {tot }}=$ Número total de enlaces de péptidos en el sustrato de la proteína $(\mathrm{eq} / \mathrm{Kg}) ; \mathrm{V}_{\mathrm{NaOH}}=$ Volumen de $\mathrm{NaOH}$ total $(\mathrm{L}), \mathrm{N}_{\mathrm{b}}=$ Normalidad de la base, $\mathrm{Mp}=$ masa de la proteína (en $\mathrm{kg}$ ), GH = grado de hidrolisis.

El grado de disociación de los grupos a-NH2 liberados en la reacción, a, se calcula directamente de la ecuación (2), y depende del pH de trabajo y del pK. Este último varía de forma significativa con la temperatura de reacción y puede estimarse de acuerdo con la ecuación (3) (Salazar et al., 2012). Se empleó un a calculado de 0,992 y un htot de $8,8 \mathrm{meq} / \mathrm{g}$ que ha sido reportado para proteínas del lactosuero (Adler-Nissen, 1986).

$$
\begin{aligned}
& \propto=\frac{1}{1+10^{\mathrm{pK}-\mathrm{pH}}} \\
& \mathrm{pK}=7,8+\frac{298-\mathrm{T}}{298 \times \mathrm{T}} \times 2400
\end{aligned}
$$

Dónde: $\alpha$ = Grado de disociación de la proteína y T= temperatura de trabajo en grados Kelvin.

\section{Descripción del diseño}

En el análisis se verificó la influencia de $E_{0}(150,300$ y $600 \mathrm{mg} / \mathrm{L})$ y de $S_{0}(5,10,10,20$ y 20,40 $\mathrm{g} / \mathrm{L})$ a través del tiempo de reacción (0-120 min), sobre la capacidad antioxidante in-vitro (ABTS y FRAP) y la capacidad quelante de hierro (CQFe). Se realizó un análisis comparativo de la evolución de las distintas variables dependientes en dos grupos de experimentos: $A$ ) variando $S_{0}$ y manteniendo constante $E_{0}$; B) manteniendo constante $S_{0}$ y variando $E_{0}$, según se indica en la tabla 1. Los niveles de la concentración de sustrato coinciden con la región cercana al valor de Km, donde la velocidad inicial de hidrólisis es considerable, en la curva de saturación. Mientras que los niveles de enzima empleados fueron definidos por ensayos previos no publicados. En el análisis, se utilizó un ANOVA para medidas repetidas con un factor intra-sujeto (tiempo: 0 , 10, 20,75 y $120 \mathrm{~min}$ ) y un factor inter-sujeto ( $\mathrm{S}_{0}$ ó $\mathrm{E}_{0}$ para cada experimento), con nivel de significación estadística de 0,05. Cada hidrolisis a las condiciones fijadas por el diseño se realizó por triplicado.

\section{Potencial Bioactivo del hidrolizado}

Se tomaron muestras en los tiempos indicados, lo cual representa distintos grados de hidrolisis. Las muestras se sometieron a $90^{\circ} \mathrm{C}$ por 10 min para la desactivación enzimática y luego se congelaron a $-20^{\circ} \mathrm{C}$ para su posterior uso en los análisis in -vitro de las actividades antioxidantes evaluadas. 
Tabla 1: Condiciones de trabajo (niveles de variables de control y variables dependientes) para los experimentos realizados : A) sustrato variable y $B$ ) enzima variable.

\begin{tabular}{|c|c|c|c|c|c|c|c|}
\hline \multirow{4}{*}{$\begin{array}{c}\text { Experimento A) } \\
E_{0}(300 \mathrm{mg} / \mathrm{L})\end{array}$} & \multicolumn{2}{|c|}{$S_{0}(g / L)$} & \multicolumn{4}{|c|}{ Factor intra-sujeto tiempo (min) } & \multirow[t]{4}{*}{ Variables dependientes } \\
\hline & Bajo & 5,11 & \multirow{7}{*}{10} & \multirow{7}{*}{20} & \multirow{7}{*}{75} & \multirow{7}{*}{120} & \\
\hline & Medio & 10,22 & & & & & \\
\hline & Alto & 20,44 & & & & & \\
\hline \multirow{4}{*}{ So $(10,22 \mathrm{~g} / \mathrm{L})$} & \multicolumn{2}{|c|}{$E_{0}(\mathrm{mg} / \mathrm{L})$} & & & & & \multirow[t]{4}{*}{ ABTS; FRAP } \\
\hline & Bajo & 150 & & & & & \\
\hline & Medio & 300 & & & & & \\
\hline & Alto & 600 & & & & & \\
\hline
\end{tabular}

Determinación de actividad antioxidante por ABTS

Se realizó siguiendo el método descrito por Re et al. (1999), en el cual $100 \mu \mathrm{L}$ de la muestra o estándar Trolox, se mezclaron con $1000 \mu \mathrm{L}$ de la solución ABTS. Luego se incubaron a temperatura ambiente por 30 minutos. La absorbancia se registró a 730nm en un espectrofotómetro UV-Vis Genesys ${ }^{\mathrm{TM}}$ 10S UV-Vis (Thermo Scientific, EE.UU.), empleando solución de Trolox de concentraciones 0- $200 \mu \mathrm{M}$ como patrón. Los resultados se expresaron como micromol de equivalentes Trolox por gramo de proteína ( $\mu \mathrm{mol}$ ET/g).

\section{Determinación de actividad antioxidante FRAP}

Se determinó por la metodología descrita por Pulido et al. (2000), $900 \mu \mathrm{L}$ del reactivo FRAP (con TPTZ, FeCl 3 y tampón acetato $3 \mathrm{M}$ ), se mezclaron con $90 \mu \mathrm{L}$ de agua destilada y $30 \mu \mathrm{L}$ de la muestra o estándar Trolox y se incubaron a $37^{\circ} \mathrm{C}$ en un baño seco por 30 min. Se midió el incremento en la absorbancia a $595 \mathrm{~nm}$, empleando un espectrofotómetro Genesys ${ }^{\mathrm{TM}}$ 10S UV-Vis (Thermo Scientific, EE.UU.), contra un blanco de buffer acetato de sodio. Se empleó una solución de Trolox de concentraciones 0-300 $\mu \mathrm{M}$ como patrón. Los resultados se expresan como micromol de equivalentes Trolox por gramo de proteína ( $\mu \mathrm{mol} \mathrm{ET} / \mathrm{g}$ proteina).

\section{Actividad quelante de hierro}

Se empleó el método descrito por Decker y Welch (1990), con algunas modificaciones. Se mezcló $1 \mathrm{~mL}$ de la muestra con $20 \mu \mathrm{l}$ de una solución de $\mathrm{FeSO} 4 \cdot 7 \mathrm{H}_{2} \mathrm{O} 2 \mathrm{mM}$. La mezcla se agitó y dejó en reposo durante 5 minutos. Se adicionó $40 \mu \mathrm{l}$ de una solución de ferrozina $5 \mathrm{mM}$ y se agitó vigorosamente. Se esperó por $10 \mathrm{~min}$ y se leyó la absorbancia a $562 \mathrm{~nm}$. El porcentaje de quelación se calculó de acuerdo a la ecuación 4.

$$
\% \text { Quelación }=\left[\frac{A B S \text { control-ABS muestra }}{A B S \text { control }}\right] \times 100
$$

\section{Purificación de fracciones peptídicas por ultra centrifugación (UF)}

Los hidrolizados de lactosuero que mostraron mayor actividad antioxidante se concentraron por ultracentrifugación utilizando tubos de ultrafiltración Amicon Ultra-15 (Merck Millipore, Alemania), con membranas PLHK Ultracel-PL, con volumen de $15 \mathrm{~mL}$ y con cortes de peso molecular de 100, 10 y $3 \mathrm{kDa}$. Se centrifugaron a 5000 x g durante 20 min en una centrifuga Modelo U- 320 (BOECO, Hamburg, Alemania). Se obtuvieron cuatro fracciones ( $>100 \mathrm{kDa} ; 10-100 \mathrm{kDa} ;<10 \mathrm{kDa}$ y $<3 \mathrm{kDa}$ ), las cuales, luego de separadas, fueron almacenadas $\mathrm{a}-20^{\circ} \mathrm{C}$, para análisis posteriores. La actividad antioxidante de las fracciones fue nuevamente determinada mediante métodos de FRAP y ABTS.

\section{RESULTADOS Y DISCUSIÓN}

A continuación, se presentan los resultados del comportamiento cinético, el diseño experimental y las actividades en función del tiempo, para diferentes concentraciones de enzima y de sustrato.

Cinética de hidrolisis enzimática de LSB-Alcalasa $\circledast 2.4 \mathrm{~L}$

La constante de afinidad $\left(\mathrm{K}_{\mathrm{m}}\right)$ y la velocidad máxima $\left(\mathrm{V}_{\max }\right)$ para el sistema de reacción $L S B$ - Alcalasa $\circledast 2.4$ $L$, se determinaron a través del análisis de saturació de la enzima en un rango amplio de $S_{0}(1-32 \mathrm{~g} / L)$, utilizando una concentración de enzima fija de $225 \mathrm{mg} / \mathrm{L}$, empleando los valores de velocidad inicial, estimadas a través de la pendiente de la curva de concentración (mM) de enlaces aminos hidrolizados ( $\alpha-\mathrm{NH})$ y determinados por método $\mathrm{pH}$-Stat, contra el tiempo (min). Cada curva fue realizada por duplicado. 


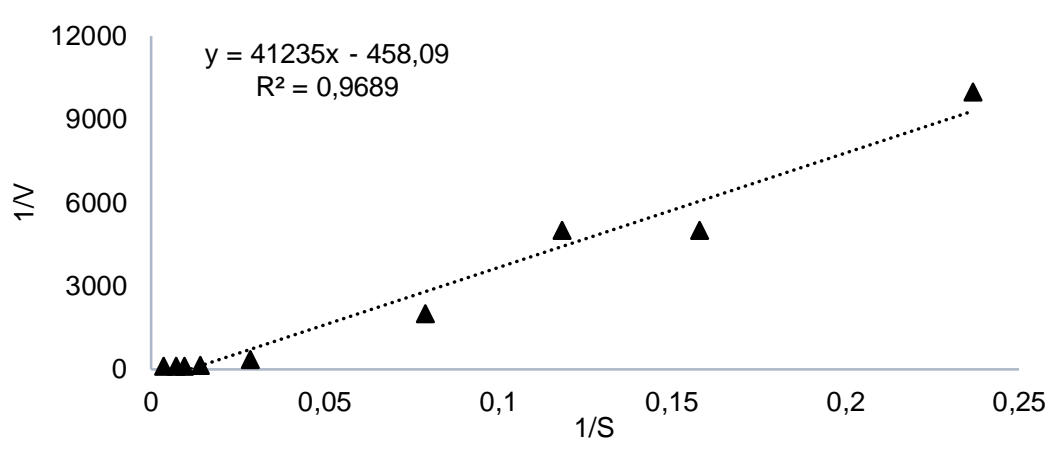

Fig. 2: Representación gráfica de inversos de velocidad inicial a distintas concentraciones de sustratos (1-32 g/L) según Lineweaver-Burk.

Los resultados registran un comportamiento típico para un sistema de saturación enzimática sin evidencia de efectos de inhibición por sustrato en los rangos de la concentración evaluada, tal como se muestra en la gráfica de dobles recíprocos de Lineweaver-Burk (Fig. 2). Para el sistema de reacción LSB- Alcalasa® 2.4 L, se obtuvo un valor de $V_{\max }$ igual a $0,00218 \mathrm{mmol} / \mathrm{min}$ y un $\mathrm{K}_{\mathrm{m}}$ con valor de $10,22 \mathrm{~g} / \mathrm{L}$, los cuales fueron obtenidos con un coeficiente de determinación significativo $\left(R^{2}=0,968\right)$.

\section{Efecto de la enzima y el sustrato en el potencial antioxidante}

El valor del $\mathrm{Km}$ representa una medida de afinidad entre Alcalasa $\AA^{8} 2.4 \mathrm{~L}$ y el sustrato, esta concentración de sustrato se tomó como referencia para analizar el efecto de la concentración de enzima sobre la actividad antioxidante (FRAP y ABTS) en distintos tiempos de hidrólisis (Exp B).

En la tabla 2 se muestran los p-valores para las distintas pruebas estadísticas. En los resultados del análisis multivariado, los cuatro estadísticos: Traza de Pillai, Lambda de Wilks, Traza de Hotelling y Raíz mayor de Roy, indican que el factor tiempo es significativo $(p<0,005)$, tanto para los valores de la actividad antioxidante de ABTS como para FRAP. Lo mismo acurre con la interacción Tiempo* $\mathrm{E}_{0}$ para la actividad medida con FRAP. Sin embargo, solo la Raíz mayor de Roy indica significancia estadística para esta interacción, en el caso de los resultados de ABTS. Puesto que el nivel crítico para la prueba de esfericidad de "W de Mauchly" resultó mayor que 0,05 para los dos casos, podemos asumir esfericidad y por lo tanto analizar los resultados de los efectos del factor intra-sujeto: tiempo, sobre la actividad antioxidante medida por los métodos FRAP y ABTS, utilizando la aproximación univariada. Como se evidencia en la tabla 2, existe un efecto individual del tiempo y su interacción con los niveles de enzima empleados sobre la respuesta evaluada (potencial antioxidante), tanto si asumimos esfericidad como utilizando dos de los correctores épsilon (Greenhouse-Geisser y HuynhFeldt), pues en todos los casos se obtienen $p$-valores $<0,030$. En lo referido al factor inter-sujeto: concentración de enzima inicial añadida $\left(E_{0}\right)$, en los niveles evaluados, los resultados indican que tanto la actividad FRAP ( $p$-valor $<0,001$ ), como la ABTS ( $p$-valor $<0,025)$, son distintas en alguno de los niveles analizados.

En el análisis de comparaciones múltiples el nivel crítico ajustado con la corrección de Bonferroni, indica que en general, la respuesta de la actividad antioxidante medida por ABTS, es significativamente $(p<0,0020)$ mayor a los $120 \mathrm{~min}$ que a los $10 \mathrm{~min}$, a pesar que ya a este tiempo, se ha alcanzado en promedio el $50 \%$ del GH total para cada nivel de enzima analizado. El análisis de la evolución de la actividad ABTS entre pares del factor $E_{0}$, muestra que solo hasta los 75 min de reacción se muestra una diferencia entre los niveles bajos y altos $(p<0,012)$, y medios y altos $(p<0,026)$ de enzima evaluados, aunque no parece existir diferencias entre los GHs alcanzados $(p<0,05)$. En contraste, los valores de FRAP comienzan a ser sustancialmente distintos en los distintos niveles de enzima, desde los $10 \mathrm{~min}$ de hidrolisis, siendo significativamente distintos entre cada uno de los tres niveles para el final de la reacción (120 min), cuando el GH es en promedio de 19\%.

Para el caso de los cambios en los niveles de sustrato, tanto el análisis multivariado como el univariado, concuerdan en que el tiempo tiene efecto significativo sobre las actividades antioxidantes FRAP y ABTS. Sin embargo, la interacción del tiempo con el sustrato no parece tener efecto, según lo indican los valores-p del experimento $A$ en tabla 1. Comparaciones múltiples muestran, que la actividad ABTS medida, es contundentemente diferente $(p<0,002)$ en todas las combinaciones de niveles de sustrato, en todos niveles de tiempo, con excepción del nivel medio y alto de sustrato a los $75 \mathrm{~min}$, donde el GH registrado es diferente. Un resultado similar ocurre con los valores de las comparaciones de las medias marginales a los 75 minutos para FRAP, donde no parece existir diferencias entre los niveles de esta actividad en los distintos niveles de sustrato empleado. Sin embargo, es importante tener en cuenta que unas de las mayores variabilidades encontradas, se registraron precisamente en estas muestras (coeficiente de variación >20\%). 
Tabla 2: ANOVA de medidas repetidas en el tiempo para los experimentos con sustrato variable (Exp A) y enzima variable (Exp B), con capacidad ABTS y FRAP como respuesta.

\begin{tabular}{|c|c|c|c|c|c|c|c|c|c|c|}
\hline \multicolumn{6}{|c|}{ Analisis Multivariado } & \multicolumn{5}{|c|}{ Analisis Univariado } \\
\hline \multirow{2}{*}{ Factor } & \multirow{2}{*}{ TEST } & \multicolumn{2}{|c|}{ ABTS (p-valor) } & \multicolumn{2}{|c|}{$F R A P(p$-valor $)$} & \multirow[t]{2}{*}{ TEST } & \multicolumn{2}{|c|}{$A B T S$ (p-valor) } & \multicolumn{2}{|c|}{ FRAP(p-valor) } \\
\hline & & $\operatorname{Exp} A$ & $\operatorname{Exp} B$ & $\operatorname{Exp} A$ & $\operatorname{Exp} B$ & & $\operatorname{Exp} A$ & $\operatorname{Exp} B$ & $\operatorname{Exp} A$ & $\operatorname{Exp} B$ \\
\hline \multirow{4}{*}{ Tiempo } & Traza de Pillai & 0,002 & 0,005 & 0,015 & 0,004 & $\begin{array}{l}\text { Esfericidad } \\
\text { asumida }\end{array}$ & 0,000 & 0,001 & 0,000 & 0,001 \\
\hline & $\begin{array}{l}\text { Lambda de } \\
\text { Wilks }\end{array}$ & 0,002 & 0,005 & 0,015 & 0,004 & $\begin{array}{l}\text { Greenhouse- } \\
\text { Geisser }\end{array}$ & 0,001 & 0,004 & 0,000 & 0,009 \\
\hline & $\begin{array}{l}\text { Traza de } \\
\text { Hotelling }\end{array}$ & 0,002 & 0,005 & 0,015 & 0,004 & Huynh-Feldt & 0,000 & 0,001 & 0,000 & 0,001 \\
\hline & $\begin{array}{l}\text { Raíz mayor de } \\
\text { Roy }\end{array}$ & 0,002 & 0,005 & 0,015 & 0,004 & & & & & \\
\hline \multirow{4}{*}{$\begin{array}{c}\text { Tiempo* } \\
\text { EO }\end{array}$} & Traza de Pillai & 0,003 & 0,087 & 0,323 & 0,021 & $\begin{array}{l}\text { Esfericidad } \\
\text { asumida }\end{array}$ & 0,000 & 0,014 & 0,096 & 0,001 \\
\hline & $\begin{array}{l}\text { Lambda de } \\
\text { Wilks }\end{array}$ & 0,004 & 0,052 & 0,318 & 0,002 & $\begin{array}{l}\text { Greenhouse- } \\
\text { Geisser }\end{array}$ & 0,001 & 0,032 & 0,114 & 0,010 \\
\hline & $\begin{array}{l}\text { Traza de } \\
\text { Hotelling }\end{array}$ & 0,008 & 0,052 & 0,365 & 0,001 & Huynh-Feldt & 0,000 & 0,014 & 0,096 & 0,001 \\
\hline & $\begin{array}{l}\text { Raíz mayor de } \\
\text { Roy }\end{array}$ & 0,002 & 0,008 & 0,081 & 0,000 & & & & & \\
\hline
\end{tabular}

Es conocido que en la hidrólisis con Alcasa 2,4 L se favorece la capacidad antioxidante de proteínas. Como se ha evidenciado en numerosos trabajos, el GH aumenta con el tiempo y del mismo modo la capacidad antioxidante mejora (Bah et al., 2015). Esto, debido a que la actividad antioxidante es principalmente atribuida a péptidos de tamaño molecular bajo y en alguna forma, el GH se ha generalizado como una herramienta indirecta para describir la distribución del peso molecular de los hidrolizados, en tanto que, una hidrólisis prolongada redunda casi siempre en grados de hidrolisis altos y por tanto tamaños peptídicos mucho más pequeños (Morales et al., 2017). Los resultados son coherentes con este análisis, sin embargo, los datos concuerdan en que esta actividad antioxidante no es necesariamente la misma para fracciones con GHs similares, logrados con combinaciones de niveles distintos.

\section{Actividad antioxidante y GHs en reacciones con sustrato y enzima variada}

Existe la tendencia global a aumentar la actividad antioxidante in -vitro en la medida que la hidrólisis avanza, con grandes diferencias en los moles de equivalente trolox registrados por las técnicas FRAP y ABTS. En tanto, es conocido que las fuentes alimenticias sean menos propensas a la reducción del ion $\mathrm{Fe}^{+3}$, que a captar radicales ABTS y peroxilo (Gómez et al., 2013). Como se muestra en las figuras 3 (A y B), actividades importantes se registran para los tiempos mayores, lo que concuerda con GHs entre los $15-20 \%$. Sin embargo, se evidencia una mayor actividad antioxidante cuando se emplean menores concentraciones de enzima, en comparación con la mayor cantidad usada, para los tiempos de 75 y 120 min, muy a pesar de que los GHs son significativamente distintos para estos dos tiempos en cada nivel de enzima analizado (GHs: $\mathrm{E}_{0}$ Baja $=$ $15,70 \%$ y 18,50 \%; E0 Alta 17,70 \% y 19,70\%; respectivamente). En estos tiempos de hidrólisis no se evidencia diferencia en términos de ABTS para los niveles medios y bajos de enzima. Para GHs iguales o superiores $16 \%$, los valores de actividad antioxidante ABTS son del orden de los 1000 o más $\mu$ molTE por gramo de proteína, en especial cuando se usan los niveles bajos y medio de enzima, lo cual significa emplear entre el 1,5-3 \% de enzima en relación con la masa de proteína total. De manera similar ocurre con los resultados de FRAP en la Fig. 3B, de nuevo las mayores actividades se registran desde los 75 min de hidrólisis para el menor nivel de enzima, sin diferencias con el nivel medio, pero significativamente distintas del nivel alto.

De otro lado, cuando se realizan cambios en la concentración de sustrato inicial de operación, se puede notar que las actividades antioxidantes ABTS y FRAP tienen comportamientos distintos, como se muestra en las figuras 4 ( $\mathrm{A}$ y B). Para el caso de ABTS, desde los 75 min se evidencian los $\mu$ molTE por gramo de proteína (superiores a 800) sin diferencias entre los niveles medio y alto de sustrato (Fig. 4A). Es decir, que la operación con concentraciones bajas de sustrato a pesar de exhibir una evolución en GHs con el tiempo relativamente mayor e incluso alcanzar GHs finales mayores, no resulta satisfactoria para la obtención de fracciones peptídicas con capacidad para neutralizar cationes ABTS ${ }^{+}$. Para el caso de FRAP de la Fig. 4B, se puede observar que todos los hidrolizados alcanzados con el mayor nivel de sustrato y los correspondientes a los tiempos finales, en los niveles medios y bajos, resultaron mayores de $400 \mu$ molTE por gramo de proteína; valores satisfactorios, si consideramos que el método no mide los antioxidantes que contienen grupos $\mathrm{SH}$, tal como algunos aminoácidos, ya que estos no reducen de forma efectiva el $\mathrm{Fe}^{3+} \mathrm{a} \mathrm{Fe}^{2+}$. 

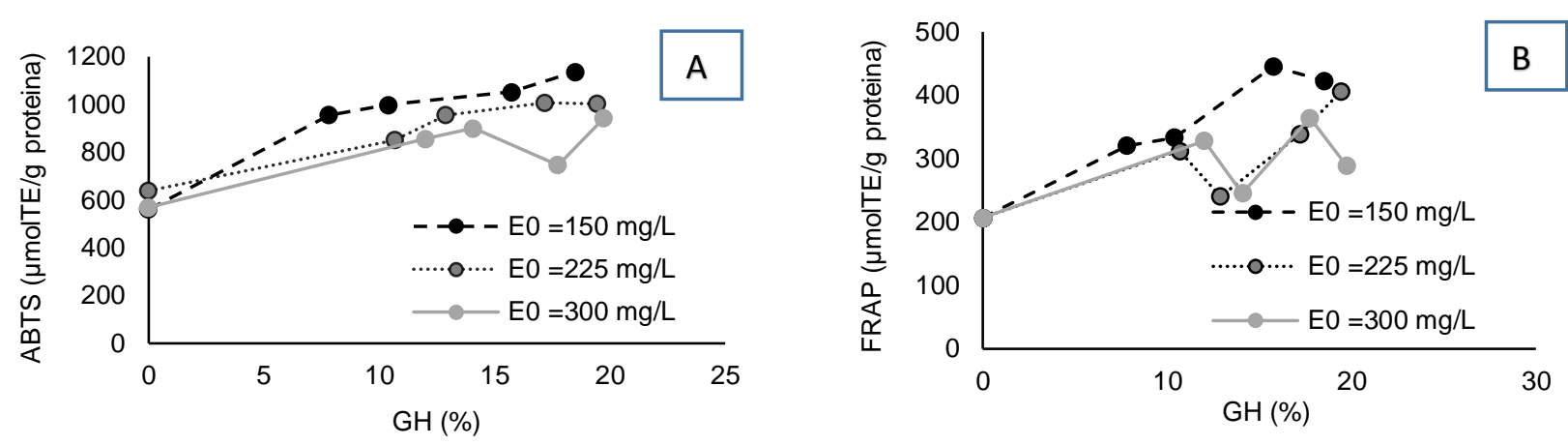

Fig. 3: Actividad antioxidante medida por (A) ABTS y (B) FRAP en lactosuero bovino para diferentes concentraciones de enzima $\left(E_{0}\right)$ Vs. GH, con $S_{0}$ de $10,22 \mathrm{~g} / \mathrm{L}$.

En los estudios de Seo et al. (2015), la habilidad para captar radicales libres de los hidrolizados de proteína del plasma se explica a través de varios mecanismos: la capacidad de donar hidrógeno, estabilizar radicales, secuestrar iones metálicos prooxidativos y probablemente formar una barrera física alrededor de las gotas de grasa usando un aminoácido particular. Según Saiga et al. (2003), esta capacidad antioxidante está relacionada con la existencia de varios aminoácidos como la tirosina, triptófano, metionina, lisina, histidina y cisteína; sin embargo, no solo la presencia de estos aminoácidos puede ser suficiente para exhibir la actividad, sino que también influyen la estructura primaria de los péptidos y su secuencia de aminoácidos.
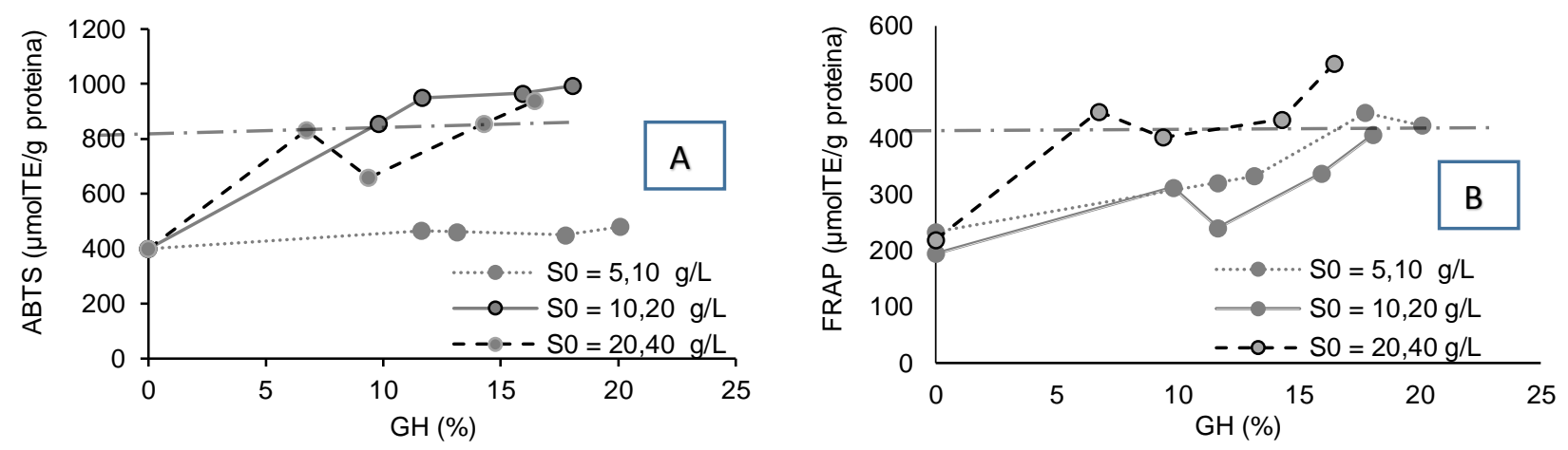

Fig.4. Tendencia de actividad antioxidante medida mediante (A) ABTS y (B) FRAP en lactosuero bovino para diferentes concentraciones de sustrato (SO) Vs. GH, con $\mathrm{E}_{0}$ de $300 \mathrm{mg} / \mathrm{L}$.

Evidentemente muchos de los aminoácidos que conforman la estructura proteica, pueden ejercer efectos antioxidantes específicos, pero dentro de la cadena proteica su actividad puede estar limitada por dificultades de accesibilidad de los oxidantes. Por otro lado, se conoce que los aminoácidos libres no son efectivos antioxidantes y aunque la hidrólisis enzimática puede mejorar la capacidad antioxidante de proteínas, una excesiva hidrólisis puede resultar negativa (Elias et al., 2008). Por ejemplo, la degradación proteolítica de la albúmina de suero bovino produce radicales proteicos con vidas medias más largas en relación con la albúmina de suero bovino nativo, mientras que la proteólisis extensa produce radicales BSA de corta duración (Østdal et al., 1999). Pietraforte y Minetti (1997) citado en Elias et al. (2008), señalan que los radicales tirosilo se detectan en péptidos que contienen tirosina después del tratamiento con peroxinitrito únicamente si el residuo de tirosina se encuentra en una posición central de una secuencia peptídica relativamente larga. De esta manera, la mayor capacidad de los hidrolizados de proteínas para disminuir la reactividad de un radical libre está relacionada con un aumento en la exposición de los aminoácidos, lo que conduce a un incremento de las reacciones de radicales libres con el péptido y en tanto potencia su efecto antioxidante.

Butré et al. (2012), encontraron que, para hidrolizados con los mismos GHs, los hidrolizados de $1 \%$ de lactosuero bovino contienen menos proteína intacta que los hidrolizados producidos con más altas concentraciones de sustrato, debido a que la afinidad de la enzima hacia la proteína la determina el equilibrio entre la proteína nativa y la desplegada en solución (Deng et al., 2018). Cambios en este equilibrio pueden sugerir cambios en los mecanismos de hidrolisis. Se ha demostrado que la afinidad de la proteína intacta fue diferente en la hidrólisis con Alcalasa para distintas concentraciones de sustrato, es decir, la composición de estos hidrolizados fue distinta y en efecto esto se produjo por cambios en la selectividad de la enzima en condiciones variadas de operación (Butré et al., 2012). Esto puede explicar por un lado las diferencias expuestas en las actividades antioxidantes medidas para distintos GHs en niveles distintos de sustrato. 


\section{Actividad quelante de hierro}

En la figura 5, se muestran los resultados del porcentaje de quelación contra el GH, para la reacción empleando $10,22 \mathrm{~g} / \mathrm{L}$ de sustrato y $300 \mathrm{mg} / \mathrm{L}$ de enzima. Se observa que los hidrolizados de LSB presentan altos porcentajes de quelación de $\mathrm{Fe}^{+}$en general. Es conocido que la actividad quelante mejora no significativamente con el aumento del GH. Sin embargo, cuando la hidrólisis enzimática no es prolongada y en donde se espera que estén presentes péptidos de tamaño intermedio y proporciones menores de péptidos de peso molecular bajo, se obtuvieron altos porcentajes de quelación de $\mathrm{Fe}^{+}(87,92 \%)$. En efecto, muchos grupos funcionales en una proteína pueden contribuir a la capacidad para fijar metales. Cadenas laterales de residuos de aminoácidos dentro de estructuras peptídicas como cisteína, serina, histidina, el aspartato y el glutamato pueden servir como ligandos para la unión de metales de transición. En principio se ha relacionado el potencial para ligar hierro a hidrolizados de bajo peso molecular, por ejemplo, Eckert et al. (2014), evaluaron el efecto del tamaño de fracciones peptídicas, separando el hidrolizado utilizando membranas y con varias proteasas, las fracciones menores de $1 \mathrm{kDa}$ pueden ser más activas que las mayores. Sin embargo, existe evidencia de que las proteínas hidrolizadas, fracciones y péptidos de diferentes tamaños mejoran el efecto sobre la absorción y biodisponibilidad de calcio y hierro (Walters et al., 2018). En contraste, Tang et al. (2009), observaron que en hidrolizados de proteína de cáñamo un aumento de la longitud de la cadena peptídica podría conducir a mayores efectos quelantes de hierro. Las fuertes propiedades quelantes de metales de los péptidos de cadena larga pueden deberse a los efectos sinérgicos de un mayor número de residuos de aminoácidos en comparación con los péptidos más cortos.

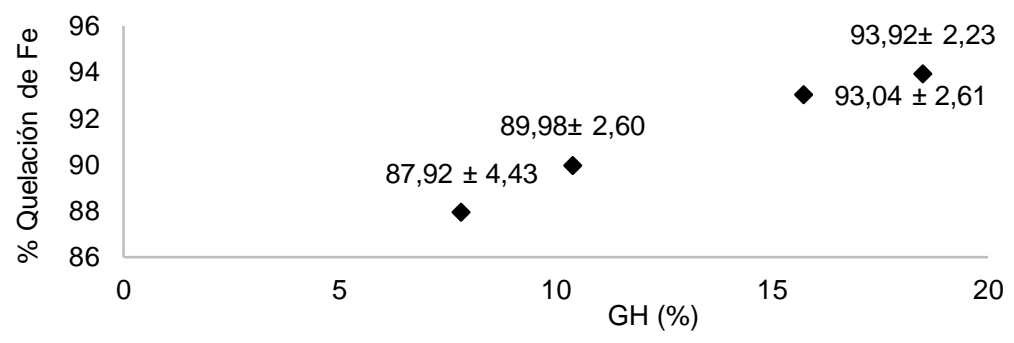

Fig. 5. Porcentaje de quelación Vs. \% GH. Concentración de sustrato 10,22 g/L, empleando 300 mg/L de enzima

\section{Concentración de fracciones peptídicas antioxidantes}

En la tabla 3, se muestra los datos de equivalentes trolox medidos a las fracciones en una serie de tamaños distintos. Los datos corresponden a hidrolizados obtenidos con el nivel medio de sustrato: 10,22 g/L y los niveles bajos y medios de enzima empleados en el experimento "B" de la tabla 1. Los resultados de los ensayos de ABTS sugieren que la fracción con péptidos menores a $3 \mathrm{kDa}$ tienen un efecto reductor de radicales libres significativamente mayores, seguido de la fracción menores de $10 \mathrm{KDa}$, con diferencias significativas $(p<0,05)$. Peng et al. (2009), obtuvieron resultados similares en hidrolizados de aislado de proteína de suero con Alcalasa. La fracción con péptidos en el rango de 0,1 a 2,8 kDa mostró una mayor actividad antioxidantes que las fracciones con péptidos de mayor tamaño. Por otro lado, los ensayos con FRAP sugieren que la fracción entre $10 \mathrm{KDa}$ y $100 \mathrm{KDa}$ son las que mayor actividad antioxidante exhiben y no existe diferencia significativa entre las otras tres fracciones. Peña, et al. (2004) encontraron que en los hidrolizados de proteína de suero están presentes fracciones peptídicas que pueden llegar a ser tanto antioxidantes como pro-oxidantes, por lo que el efecto esperado como antioxidante se ve reducido por el efecto antagonista. En general la habilidad antioxidante de los péptidos de lactosuero se relaciona con estructura de estos, en tanto que residuos de aminoácidos, tales como metionina, histidina, lisina y triptófano, presentes en la secuencia de péptidos de la $\beta$-Lactoglobulina, los cuales tienen la capacidad de quelar iones metálicos pro-oxidantes (Gurrola et al., 2017).

Tabla 3: Comparación entre grupos de fracciones obtenidas por ultrafiltración despues de hidrolisis con $\mathrm{S}_{0}=10,22 \mathrm{~g} / \mathrm{L}$ y y los niveles bajos y medios de enzima empleados en el experimento "B

\begin{tabular}{|c|c|c|c|c|}
\hline \multirow{2}{*}{ Fracciones } & \multicolumn{2}{|c|}{ FRAP $\mu \mathrm{mo} / \mathrm{TE} / \mathrm{g}$} & \multicolumn{2}{c|}{ ABTS $\mu \mathrm{mo} / \mathrm{TE} / \mathrm{g}$} \\
\cline { 2 - 5 } & $E_{0=150 \mathrm{mg} / \mathrm{L}}$ & $E_{0}=300 \mathrm{mg} / \mathrm{L}$ & $E_{0=150 \mathrm{mg} / \mathrm{L}}$ & $E_{0=300 \mathrm{mg} / \mathrm{L}}$ \\
\hline$>100 \mathrm{kDa}$ & $241,08 \pm 15,90$ & $285,09 \pm 61,46$ & $771,35 \pm 18,26$ & $669,79 \pm 28,88$ \\
\hline $10-100 \mathrm{Kda}$ & $304,99 \pm 35,57$ & $313,08 \pm 28,32$ & $804,69 \pm 55,64$ & $707,23 \pm 98,50$ \\
\hline$<10 \mathrm{kDa}$ & $273,06 \pm 29,39$ & $259,82 \pm 30,26$ & $965,62 \pm 28,62$ & $918,46 \pm 95,96$ \\
\hline$<3 \mathrm{kDa}$ & $282,03 \pm 24,43$ & $277,32 \pm 20,70$ & $1038,55 \pm 48,02$ & $1080,98 \pm 51,67$ \\
\hline
\end{tabular}




\title{
CONCLUSIONES
}

En la reacción de hidrólisis enzimática de LSB con Alcalasa existe un efecto significativo del tiempo sobre el potencial antioxidante de los hidrolizados de lactosuero. Además, la actividad antioxidante es mayor a menores concentraciones de enzima. Con distintos niveles de enzima, el incremento en el GH favorece la actividad antioxidante medida por el método ABTS así como la actividad quelante de metales de los hidrolizados. Por otra parte, en la operación de éste sistema de reacción, con concentraciones bajas de sustrato, a pesar de presentar mejor comportamiento en términos de GHs con el tiempo, alcanzando incluso GHs finales relativamente superiores a los obtenidos con otros niveles de sustrato, las fracciones peptídicas obtenidas no poseen gran potencial en la capacidad para neutralizar cationes ABTS ${ }^{+}$. Para el caso de la actividad medida por FRAP, todos los hidrolizados alcanzados con el mayor nivel de sustrato y los correspondientes a los tiempos finales, en los niveles medios y bajos, resultaron mayores de $400 \mu \mathrm{molTE}$ por gramo de proteína, lo cual es satisfactorio.

\section{AGRADECIMIENTOS}

Agradecemos al Grupo de Nutrición y Tecnología de Alimentos de la Universidad de Antioquia y a la Universidad Popular del Cesar por el apoyo financiero y logístico que han hecho posible este trabajo.

\section{NOTACIÓN}

\author{
Abreviaciones \\ $\mathrm{AOAC}=$ Association of Official Analytical Chemists. \\ FRAP = poder antioxidante de la reducción férrica. \\ ABTS = azinobis 3-etilbenzotiazolina-6-ácido sulfónico. \\ Súper/sub índices \\ max = máximo
}

\section{REFERENCIAS}

Adler-Nissen, J., Enzymic hydrolysis of food proteins, $1^{\text {a }}$ Ed., 365 -404, Elsevier applied science publishers, Londres, Inglaterra (1986).

Bah, C.S. Bekhit, A.E. y otros dos autores, Production of bioactive peptide hydrolysates from deer, sheep and pig plasma using plant and fungal protease preparations, doi: 10.1016/j.foodchem.2014.12.025, Food Chem, 176, 54-63 (2015).

Butré, C.L. Wierenga, P.A. y Gruppen, H., Effects of ionic strength on the enzymatic hydrolysis of diluted and concentrated whey protein isolate, doi: 10.1021/jf301409n, J. Agric. Food Chem, 60(22), 5644-5651 (2012).

De Castro, R.J.S. y Sato, H.H., Biologically active peptides: Processes for their generation, purification, identification, and applications as natural additives in the food and pharmaceutical industries, doi: 10.1016/j.foodres.2015.05.013, Food Res, Int. 74, 185-198 (2015).

Deng, Y. Butré, C.I. y Wierenga, P.A., Influence of substrate concentration on the extent of protein enzymatic hydrolysis, doi: 10.1016/j.idairyj.2018.06.018, Int. Dairy J, 86, 39-48 (2018).

Eckert, E. Bamdad, F. y Chen L., Metal solubility enhancing peptides derived frombarley protein, doi: 10.1016/j.foodchem.2014.03.061, Food Chem, 159, 498-506 (2014).

Elias, R.J. Kellerby, S.S. y Decker, E.A., Antioxidant Activity of Proteins and Peptides, doi: 10.1080/10408390701425615, Crit Rev Food Sci Nutr, 48. 430-441 (2008).

Fernández A. y Riera, F., $\beta$-Lactoglobulin tryptic digestion: A model approach for peptide release, doi: 10.1016/j.bej.2012.10.001, Biochem. Eng. J, 88-96 (2013).

Gómez, L. J. Figueroa, O.A. y Zapata, J.E., Actividad antioxidante de hidrolizados enzimáticos de plasma bovino obtenidos por efecto de Alcalasaß 2.4 L, doi: 10.4067/s0718-07642013000100005, Inf. Tecnol, 24(1), 33-42 (2013).

Gurrola L.R. Chávez, A., y otros dos autores, Proteínas del lactosuero: usos, relación con la salud y bioactividades, Interciencia, ISSN: 0378-1844, 42(11), 712-718 (2017).

Hinrichs, R. Götz, J. y otros cuatro autores, Characterisation of different treated whey protein concentrates by means of low-resolution nuclear magnetic resonance, doi: 10.1016/j.idairyj.2004.02.003, Int. Dairy J, 14(9), 817-827 (2004).

Marshall, K., Therapeutical applications of whey protein, Altern Med Rev, ISSN: 1089-5159, 9, 136-156 (2004).

Morales, R. Pérez, R. y otros dos autores, Multiobjective optimization of the antioxidant activities of horse mackerel hydrolysates produced with protease mixture, doi: 10.1016/j.procbio.2016.11.001, Process Biochem. 52, 149-158 (2017).

Liu, Q. Kong, B. y otros dos autores, Antioxidant activity and functional properties of porcine plasma protein hydrolysate as influenced by the degree of hydrolysis, doi: 10.1016/j.foodchem.2009.05.013, Food Chem. 118, 403-410 (2010). 
Østdal, H. Andersen, H.J. y Davies, M.J., Formation of long-lived radicals on proteins by radical transfer from heme enzymes-a common process? doi: 10.1006/abbi.1998.0988, Arch. Biochem. Biophys, 362(1), 105-112 (1999).

Peng, X. Xiong, Y.L. y Kong, B., Antioxidant activity of peptide fractions from whey protein hydrolysates as measured by electron spin resonance. Food Chemistry, 113(1), 196-201 (2009).

Peña-Ramos, E.A. Xiong, Y.L. y Arteaga, G.E., Fractionation and characterisation for antioxidant activity of hydrolysed whey protein, doi: 10.1002/jsfa. 1886, J. Sci. Food Agric, 84(14), 1908-1918 (2004).

Pulido, R. Bravo, L. Y Saura, F., Antioxidant activity of dietary polyphenols as determined by a modified ferric reducing/antioxidant power assay, doi: 10.1021/jf9913458, J. Agric. Food Chem, 48(8), 3396-3402 (2000).

Re, R. Pellegrini, N., y otros cuatro autores, Antioxidant activity applying an improved ABTS radical cation decolorization assay. doi: 10.1016/s0891-5849(98)00315-3, Free Radical Bio Med, 26 (9-10), 1231-1237 (1999).

Rutherfurd, S.M., Methodology for determining degree of hydrolysis of proteins in hydrolysates: a review, S.M.Rutherfurd@massey.ac.nz, ISSN 1944-7922J, AOAC Int, 93(5), 1515-1522 (2010).

Sabeena, K.H. Andersen, L.L. y otros cinco autores, Antioxidant activity of Cod (Gadus morhua) protein hydrolysates: In vitro assays and evaluation in 5\% fish oil-in-water emulsion, doi: 10.1016/j.foodchem.2013.03.075, Food Chem, 149, 326334 (2014).

Saidi, S. Deratani, A. y otros dos autores, Production and fractionation of tuna by-product protein hydrolysate by ultrafiltration and nanofiltration: Impact on interesting peptides fractions and nutritional properties, doi: 10.1016/j.foodres.2014.04.026, Food Res Int, 65 (C), 453-461 (2014).

Saiga, A. Tanabe, S. y Nishimura, T., Antioxidant Activity of Peptides Obtained from Porcine Myofibrillar Proteins by Protease Treatment, doi: 10.1021/jf021156g. J. Agric. Food Chem. 51 (12), 3661-3667 (2003).

Salazar, C. López, A. y Cano, J.A., Effect of the $\mathrm{pH}$ and of the temperature on the enzymatic hydrolysis of by-products from bovine industries, ISSN: 2256-3938, Rev. Lasallista Investigación, 9(2), 26-32 (2012).

Sarmadi, B.H. y Ismail, A., Antioxidative peptides from food proteins: A review, doi: 10.1016/j.peptides.2010.06.020, Peptides, 31(10), 1949-1956 (2010)

Seo, H.W. Jung, E.J. y otros cuatro autores, Optimization of hydrolysis conditions for bovine plasma protein using response surface methodology, doi: 10.1016/j.foodchem.2015.03.133, Food Chem, 185, 106-111 (2015).

Tang, C.H. Wang, X.S. y Yang, X.Q., Enzymatic hydrolysis of hemp (Cannabis sativa L.) protein isolate by various proteases and antioxidant properties of the resulting hydrolysates, doi: 10.1016/j.foodchem.2008.11.049, Food Chem, 114 (4), 1484-1490 (2009).

Valencia, P. Espinoza, K. y otros tres autores, Novel modeling methodology for the characterization of enzymatic hydrolysis of proteins, doi: 10.1016/j.procbio.2014.12.028, Process Biochem, 50, 589-597 (2015).

Walters, M. Esfandi, R. y Tsopmo, A., Potential of food hydrolyzed proteins and peptides to chelate iron or calcium and enhance their absorption, doi: 10.3390\%2Ffoods7100172, Foods, 7(10), 172 (2018).

Xie, Z. Huang, J. y otros dos autores, Antioxidant activity of peptides isolated from alfalfa leaf protein hydrolysate, doi: 10.1016/j.foodchem.2008.03.078, Food chem, 111(2), 370-376 (2008). 